Sozialistische Eliten 
Reihe „Soziologie der Politik“

Herausgegeben von

Ronald Hitzler

Stefan Hornbostel

Sighard Neckel

Band 2 
Stefan Hornbostel (Hrsg.)

\section{Sozialistische Eliten}

Horizontale und vertikale

Differenzierungsmuster in der DDR

Leske + Budrich, Opladen 1999 
Gedruckt auf säurefreiem und altersbeständigem Papier.

Die Deutsche Bibliothek - CIP-Einheitsaufnahme Sozialistische Eliten: Horizontale und vertikale Differenzierungsmuster in der DDR / Stefan Hornbostel (Hrsg.). - Opladen : Leske + Budrich, 1999

(Reihe „Soziologie der Politik“; Bd. 2)

ISBN 978-3-8100-2260-8

ISBN 978-3-322-99558-2 (eBook)

DOI 10.1007/978-3-322-99558-2

NE: Hornbostel, Stefan [Hrsg.]; GT

(C) 1999 Leske + Budrich, Opladen

Das Werk einschließlich aller seiner Teile ist urheberrechtlich geschützt. Jede Verwertung außerhalb der engen Grenzen des Urheberrechtsgesetzes ist ohne Zustimmung des Verlages unzulässig und strafbar. Das gilt insbesondere für Vervielfältigungen, Übersetzungen, Mikroverfilmungen und die Einspeicherung und Verarbeitung in elektronischen Systemen. 


\section{Inhalt}

\section{Stefan Hornbostel}

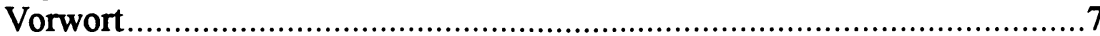

\section{Christoph Boyer}

Kaderpolitik und zentrale Planbürokratie in der SBZ/DDR

(1945-1961)

\section{Lothar Mertens}

Der Austausch einer Funktionselite

\section{Matthias Wagner}

Das Kadernomenklatursystem - Ausdruck der führenden Rolle der SED

\section{Rainer Weinert}

Die Wirtschaftsführer der SED: Die Abteilungsleiter im ZK im Spannungsfeld von politischer Loyalität und ökonomischer Rationalität

\section{Sabine Lorenz}

Kommunaler Elitenwandel: Rekrutierung, Zusammensetzung und Qualifikationsprofil des lokalen administrativen Führungspersonals in Ostdeutschland

Helga A. Welsh

Zwischen Macht und Ohnmacht: Zur Rolle der 1. Bezirkssekretäre der SED

\section{Jens Gieseke}

Die hauptamtlichen Mitarbeiter des Ministeriums für Staatssicherheit eine sozialistische Elite?

\section{Dagmar Langenhan und Sabine Roß}

Berufskarrieren von Frauen in der DDR und ihre Grenzen

Lothar Mertens und Dieter Voigt

Soziale Herkunft und Selbstrekrutierung der promovierten Intelligenz in der DDR 


\section{Stefan Hornbostel}

Die besten Vertreter der Arbeiterklasse. Kaderpolitik und gesellschaftliche Differenzierungsmuster im Spiegel des zentralen Kaderdatenspeichers des Ministerrates der DDR

Sighard Neckel

Das Sozialkapital der Survivor. Erfolgsbedingungen „persistenter“ lokaler Eliten in Ostdeutschland am Beispiel einer Gemeindestudie

\section{Hanjo Gergs und Markus Pohlmann}

Ökonomische Eliten vor und nach der Wiedervereinigung:

Die Selektivität des Transformationsprozesses.

\section{Anton Sterbling}

Elitenbildung und Elitenwandel in Südosteuropa und der ehemaligen DDR

\section{Peter Imbusch}

Politische und ökonomische Eliten in

gesellschaftlichen Transitionsprozessen. Über den Erhalt, Verlust und die Transformation von Macht. 


\section{Stefan Hornbostel}

\section{Vorwort}

Ihrem ideologisch-programmatischem Selbstanspruch zufolge war die DDR eine Gesellschaft der Gleichen. Daran änderten auch die im Rahmen sozialistischen Wettwerbs verteilten materiellen und symbolischen Gratifikation nichts. Entsprechend verpönt war der Begriff „Elite“ in der DDR; denn mit ihm waren all jene Ungleichheitsstrukturen bürgerlicher Gesellschaften assoziiert, von denen es sich abzusetzen galt. Das begriffliche Instrumentarium wie Avantgarde und Kader -, das in der DDR zur Kennzeichnung herausgehobener Leitungs- und Machtpositionen benutzt wurde, verweist bewußt auf revolutionäre Traditionen der Selektion von Führungspersonal und auf charismatische Figuren der Legitimation. Allerdings ist im Anforderungsprofil der Führungskader bereits eine Widersprüchlichkeit enthalten, die man als charakteristisch für sozialistische Gesellschaften ansehen kann, nämlich die Verbindung von fachlicher Kompetenz und Spezialistentum auf der einen Seite und politischer Loyalität, verbunden mit dem absoluten Primat des Politischen, auf der anderen Seite. Letzteres ist fast eine Chiffre für den immer wieder beschriebenen Entdifferenzierungsprozeß in der DDR, der zur Durchsetzung sehr dichter Kontrollhierarchien führte, aber die Ausdifferenzierung autonomer gesellschaftlicher Subsysteme systematisch behinderte. Spezialistentum ist hingegen ein typisches Differenzierungsphänomen, das mit dem Aufbau eigenlogisch prozessierender Teilsysteme verbunden ist. Das Führungspersonal - damit sind hier nicht nur die politische Führungsspitze, sondern auch die hohen und mittleren Leitungspositionen in Wirtschaft, Politik, Verwaltung, Kultur etc. gemeint - steht gewissermaßen im Schnittpunkt jener widersprüchlichen Anforderungsstrukturen. Das Personal auf den mittleren Führungspositionen mit seinen spezifischen Rekrutierungsmustern, Kompetenzen, Orientierungen, symbolischen Abgrenzungen und mehr oder weniger großen Handlungspielräumen bildet einen Schlüssel für das Verständnis der Funktionsweise einer sozialistischen Gesellschaft: In der Kontrollhierarchie war es in einer prekären Vermittlungsposition zwischen oben und unten placiert, in der Effizienz und Erfolge nur durch Zugeständnisse an die Eigensinnigkeit der jeweiligen Bereiche möglich waren, Aufstieg und Sicherung der eigenen Position jedoch in bisher nicht genau bekanntem Umfang durch politischen Einfluß und die Demonstration von Systemloyalität zu erreichen war. Ausgefeilte Nomenklaturen und eine extensive Kaderpolitik zeigen, daß auch in der Selbstwahrnehmung des DDR-Systems diesen mittleren Eliten große Bedeutung zugemessen wurde. 
Die in diesem Band versammelten Beiträge zielen daher bewußt nicht auf die kleine Machtelite, sondern auf den Unterbau des Systems, in dessen Struktur und Funktionsweise sich Erklärungen sowohl für die Stabilität wie auch für das Scheitern jenes gesellschaftlichen Großexperimentes „Sozialismus" finden lassen. Da die Entwicklung der DDR-Gesellschaft deutlich differente Phasen und zugehörige Generationszusammenhänge aufweist, reichen die Beiträge zeitlich von der Nachkriegszeit bis in die Nachwendezeit. Dabei kommen methodisch sehr unterschiedliche Annäherungen an den Gegenstandsbereich zum Einsatz. Auch thematisch ist ein großer Bogen gespannt, jedoch mit einem Schwerpunkt auf dem Führungspersonal in der Wirtschaft und dem politischen und administrativen Personal auf lokaler und regionaler Ebene.

Christoph Boyer untersucht für die Zeit von Kriegsende bis zum Mauerbau den konfliktreichen Formierungsprozeß, in dem jene neue „regimeeigene" Führungsschicht der DDR aufgebaut werden sollte, am Beispiel der höheren und mittleren Bürokraten in der zentralen staatlichen Wirtschaftsverwaltung. Einen wichtigen Teilaspekt des Neubeginns, nämlich den geplanten Elitenaustausch im Bildungssystem, der Zentralinstitution für die Karrierewege der neuen Elite, beleuchtet Lothar Mertens anhand der Rekrutierung der „Neulehrerschaft“. Das sich erst allmählich in seiner ganzen Komplexität entwickelnde Nomenklatursystem, also die formale Seite jener neuen geplanten und kontrollierten Elitekonfiguration, stellt Matthias Wagner in seinem Beitrag dar. Basierend auf Interviewmaterial mit Wirtschaftsführern geht Rainer Weinert der Frage nach, wieweit im weiteren Verlauf der DDR Geschichte Soll- und Ist-Strukturen auseinandergetreten sind und wie die daraus resultierenden kognitiven Dissonanzen von den Akteuren verarbeitet wurden. Sabine Lorenz widmet sich der mittleren Führungsebene und zwar dem administrativen Personal in den Kreisen und Städten der DDR. Auch hier geht es um die Austarierung von Politisierung und Verfachlichung und die Frage welche Organisationsrealität sich hinter der politischen Fassade entwickelt hat. Tertium comparationis ist dabei nicht nur die politische SollVorgabe der SED, sondern auch die Entwicklung der kommunalen Verwaltung nach der Wende. Ebenfalls um regionale Bezüge geht es Helga Welsh in ihrem Beitrag, der am Beispiel der 1. Bezirkssekretäre den Spannungen zwischen zentraler Allmacht und beschränkter regionaler Autonomie nachgeht. In einen eher klandestinen Bereich sozialistischer Elitenformation führt Jens Gieseke mit seinem Beitrag über die Entwicklung der Stasi. Da bei den MfSMitarbeitern in besonderer Weise politische Funktion und soziale Position zusammentreffen, geht es geht gleichermaßen um die spezifische Funktion der Geheimpolizei im SED-Staat wie um die gesellschaftliche Stellung und sozialstrukturelle Komposition des Personals.

Zwei klassische Ungleichheitsmuster, nämlich die Entwicklung und Perpetuierung differenter Soziallagen nach Geschlecht und Bildung, analysieren 
Dagmar Langenhan und Sabine Roß, die einer sozialistischen „Errungenschaft", nämlich der starken Einbindung der Frauen in die Arbeitswelt, nachgehen. Lothar Mertens und Dieter Voigt beleuchten die Veränderung und interne Differenzierung der Rekrutierungsmuster der „Intelligenz“. Welche generationalen, sektoralen, sozialen und geschlechtsspezifischen Rekrutierungs- und Selbstrekrutierungsmuster die mittleren und oberen Leitungsebenen der DDR-Gesellschaft entwickelten, welche politische Herkunft diese Gruppe hatte, welche politischen Bindungen sie einging und wie sie ihre interne Hierarchie symbolisch unterfütterte, zeigt der Beitrag von Stefan Hornbostel anhand einer Sekundäranalyse der routinemäßigen Selbstbeobachtungsdaten des DDR-Regimes.

Der Persistenz von Eliten auf lokaler Ebene geht Sighard Neckel in seinem Bericht aus einer Gemeindestudie in Ostdeutschland nach. Um Persistenz geht es auch im Beitrag von Hanjo Gergs und Markus Pohlmann, die die Verschränkung von Elitenzirkulation auf nationaler Ebene mit vertikaler Reproduktion auf der Ebene von regionalen Eliten und Subeliten in der Wirtschaft untersuchen. Eine Einordnung der Elitenbildung in der DDR in den südosteuropäischen Kontext unternimmt Anton Sterbling, während Peter Imbusch versucht die Implikationen drastischen sozialen Wandels auf die Elitenstruktur durch Vergleich sehr unterschiedlicher Gesellschaftssysteme darzustellen.

Alle Beiträge basieren auf Vorträgen, die Rahmen einer Tagung zum Thema „Sozialistische Eliten“ im Dezember 1997 an der Friedrich-SchillerUniversität Jena gehalten wurden. Die Durchführung der Tagung wurde durch eine Förderung des Thüringer Ministeriums für Wissenschaft, Forschung und Kultur ermöglicht, wofür an dieser Stelle gedankt sei. 